\title{
Probabilistic Opportunistic Access of Cognitive Radio Network using Partially Observable Markov Decision Process
}

\author{
Atul Singh Jamwal \\ Department of Computer Science and Engineering, \\ SVIET, Banur \\ India
}

\author{
Nidhi Bhalla \\ Department of Computer Science and Engineering, \\ SVIET, Banur \\ India
}

\begin{abstract}
Growing demands of spectrum utilization make us to think about cognitive radio. Cognitive radio is smart enough to adjust its parameters according to spectrum use thereby increasing the efficiency of spectrum access. It means it should have the ability to make optimistic decisions dynamically in a multi operated network. Partially observable markov decision process is the technique of making optimistic decisions among various alternatives. MDPs are useful for studying a wide range of optimization problems solved via dynamic programming and reinforcement learning. In this paper, we demonstrate how POMDP is useful while using cognitive radio network.
\end{abstract}

Keywords: Cognitive Radio, POMDP, spectrum heterogeneity, white Spaces

\section{INTRODUCTION}

Inefficient spectrum utilization is one of the main concerns in the field of spectrum access as most of the spectrum bandwidth remains unutilized. Moreover growing demand of spectrum access makes the situation worse. The breakneck proliferation of wireless devices and rapid growth of wireless services continue to stretch the limited spectral resource. In fact, most spectrum bands suitable for terrestrial wireless communication have already been allocated by the regulatory agencies to existing licensees. Cognitive radio networks have been considered as the viable technology to improve spectral efficiency. With primary licensee's consent, secondary users equipped with cognitive radios may be allowed [1] to transmit on primary bands when the Primary users are inactive. Serious challenges must be resolved in order for the cognitive radios to be acceptable. First, secondary users must not be disruptive to primary user communications. Secondly, an access mechanism is required to reduce contention between secondary users to efficiently share spectrum opportunities

One of the most difficult thing but important problem when designing an Open Spectrum [3] Access Network is how the unlicensed users decide when and which channel they should sense and access without conflicting the communications among Primary Users. Also, coordination and synchronization among secondary users may be limited due to the decentralized nature of secondary user access, particularly if secondary users of different networks coexist. In addition, each secondary users may not be able to sense all channels due to the limitation on hardware or/and sensing capability. Open Spectrum allows unlicensed [5] (secondary) users to share spectrum with legacy (primary) spectrum users, thereby "creating" new capacity and commercial value from existing spectrum ranges. Based on agreements and constraints imposed by primary users, secondary users opportunistically utilize unused licensed spectrum on a non-interfering or leasing basis. Open spectrum system designs must also deal with spectrum heterogeneity, where spectrum available to secondary devices fluctuates with both location and time due to movement and traffic variations of primary users. A user seizing spectrum without coordinating with others can cause harmful interference with its surrounding neighbours, thus reducing available spectrum. In open spectrum systems, primary users' mobility and traffic variations result in the fact that the available spectrum observed by secondary devices fluctuates with both location and time. We call this property spectrum heterogeneity [4]. In addition, the interference constraint and the reward (i.e. throughput, connectivity) obtained on each spectrum band could be different due to non-uniformly partitioned spectrum bands, differences in power constraints and associated technology.

\subsection{Markov Decision Process}

Markov Decision Process is a mathematical tool which provides a mathematical framework for modeling decision making in situations where outcomes are partly random and partly under the control of a decision maker. In simple terms MDP is an automation of decisions where subsequent decisions are the outcome of transition from present state to next state. While transiting from one state to next state there can be two types of outcomes: one is immediate effect and other is future gain and optimistic [9] decision is the the action that makes the right tradeoffs between the immediate rewards and the future gains, to yield the best possible solution. The four components of an MDP model are: a set of states, a set of actions, the effects of the actions and the immediate value of the actions.

- States: When making a decision, we need to think about how your actions will affect things. The state is the way the world currently exists and an action will have the effect of changing the state of the world. If we think about the set of every possible way the world could be, then this is would be the set of state of the world. Each of these states would be a state in the MDP.

- Actions: The actions are the set of possible alternatives we can make. The problem is to know which of these actions to take in for a particular state of the world.

- Transitions: When we are deciding between different actions, we have some idea of how they will affect the current state. The transitions specify how each of the actions change the state. Since an action could have different effects, depending upon the state, we need to specify the action's effect for each state in the MDP. The most powerful aspect of the MDP is that the effects of an action can be probabilistic. Imagine we are specifying the effects of doing action 'a1' in state 's1'. We could say that the effect of 'a1' is to leave the process in state 's2', if there was no question about how 'a1' changes the world. However, many decision processes have actions that are 
not this simple. Sometimes an action usually results in state 's2', but occasionally it might result in state 's3'. MDPs allow you to specify these more complex actions by allowing you to specify a set of resulting states and the probability that each state results.

- Immediate Rewards: If we want to automate the decision making process, then we must be able to have some measure of an action's value so that we can compare different actions. We specify the immediate value for performing each action in each state.

\subsection{Partially Observable Markov Decision Process}

A Partially Observable Markov Decision Process (POMDP) is a generalization of a Markov Decision Process. A POMDP models an agent [7] decision process in which it is assumed that the system dynamics are determined by an MDP, but the agent cannot directly observe the underlying state. Instead, it must maintain a probability distribution over the set of possible states, based on a set of observations and observation probabilities, and the underlying MDP.

An exact solution to a POMDP yields the optimal action for each possible belief over the world states. The optimal action maximizes (or minimizes) the expected reward (or cost) of the agent over a possibly infinite horizon. The sequence of optimal actions is known as the optimal policy of the agent for interacting with its environment

\section{POLICY STRATEGIES USED IN SPECTRUM SENSING}

Decision-making is the cognitive process leading to the selection of action among variations. One-way to automate the decision making process is to provide a model of dynamics [6] for the domain in which the machine will be making decisions. A reward structure can be used to motivate immediate decision that will maximize the future reward. POMDP is an aid in the automated decision-making. POMDP policy informs CR users what action to be executed. It can be a function or a mapping and typically depends upon the channel states. In this section, we provide detail formulation of policy strategy either optimal and sub optimal based on greedy approach for sensing decision.

- Optimal Strategy: Channel access based on POMDP is known as an optimal strategy which model the channel opportunity of network system as discrete time Markov chain with number of channel state and formulate as $M=2^{\wedge} \mathrm{N}$ states, where $\mathrm{N}$ is number of channel. The state diagram for $\mathrm{N}=2$ is described in figure 1 where state $(0,1)$ indicates the first channel is available and the second channel is busy.

- Suboptimal Strategy or Greedy Approach: Due to the complexity of optimal policy computation when number of slot and channel increase, sub optimal protocol based on a greedy approach can be used. Greedy approach follows the problem solving heuristic by making local optimal decision choices with the hope of finding the global optimum. It reduces the dimension of states from exponential to linear by regarding to $\mathrm{N}$, i.e. from $\mathrm{M}=2^{\wedge} \mathrm{N}$ to $\mathrm{N}$ states.
- Random Approach: Random Policy approach is useful for many ill-structured global optimization decision problems with continuous and/or discrete variables. Typically random policies sacrifice a guarantee of optimality for finding a good solution quickly with convergence results in probability.

\section{COMPARISON BETWEEN DIFFERENT STRATEGIES}

We have compared three different policy strategies by inputting number of channels, bandwidth and transition probabilities of each channel and time horizon $\mathrm{T}$ and obtained average expected reward per slot for each strategy. In this work we have calculated throughput performance of the greedy sensing strategy, Random sensing strategy and Optimal sensing strategy with perfect spectrum identification in independent channels as shown in figure 1.

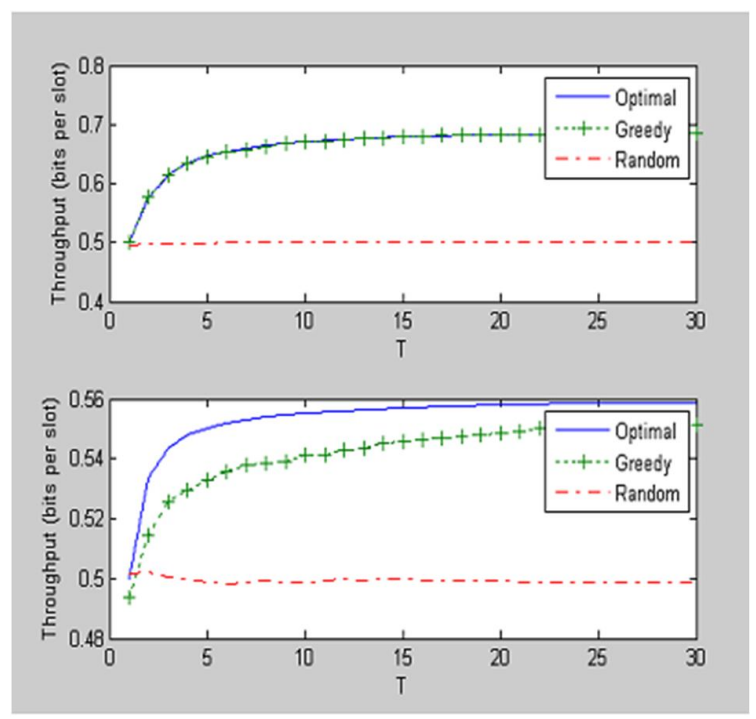

Figure 1: Comparison between different strategies

\section{CONCLUSION AND FUTURE SCOPE}

With the increasing demand for radio spectrum on one hand and inefficient usage of the licensed bands on the other, a reform of the spectrum access policy seems inevitable. Opportunistic spectrum access is envisioned to resolve the spectrum scarcity by allowing unlicensed users to dynamically utilize white spaces across the licensed spectrum on a priority basis. In this article we have implemented three decision policies to detect and sense the spectrum so as to derive an efficient decision making strategy for the effective utilization of cognitive radio technology.

The strategies discussed in this article can be used to optimize the decision making ability of the network and make it more powerful and efficient while solving dynamic decision problem which is main area of concern to implement Cognitive Radio Technology. An important venue for further research is the interplay of spectrum sensing and higher-layer functionalities to enhance the end user's perceived QoS 


\section{REFERENCES}

[1] Milind M.Buddhikot, Paul Kolodzy, Scott Miller, Kevin Ryans, Jason Evans "DIMSUMNet : New Directions in wireless networking using coordinated dynamic spectrum access", IEEE, WoWMoM05 , June 2005.

[2] I.F.Akyyilidiz , W.Y Lee , M.C.Varun , M.Shantidev "Next Generation/Dynamic Spectrum Access/ Cognitive Radios wireless Netwroks : A Survey" A Computer Network Journal(Elsevier) vol 50, Issue 13 , p2127-2159, 2006

[3] Chunyi Peng - Haitao Zheng - Ben Y. Zhao K. "Utilization and fairness in spectrum assignment for opportunistic spectrum access" Springer, May 2006 , Pg no 555-576.

[4] S.Gerihofer , L.Tong, B.M.Sadler “ Cognitive Medium Access : constraining interference based on Experimental models" IEEE journal, Selected Area communication, vol 26 No.1, pg 95-105 , 2007.

[5] Raul Etkin, Abhay Parekh, and David Tse "Spectrum Sharing for Unlicensed Bands" IEEE J.Sel area Communication. Vol.25 No.3 pp517-528, April 2007.

[6] Q.Zha , L.tong , A.Swami , Y.Chen , “ Decentralized Cognitive MAC for opportunistic Spectrum Access in Ad Hoc Networks : A POMDP Framework "IEEE J , Selected area, communication Vol.25 No.3, p589-600 , 2007.

[7] Kyounghwan Lee and Aylin Yener "Throughput Enhancing Cooperative Spectrum Sensing Strategies for Cognitive Radios” IEEE Proc of ACSSC, p2045-2049, Nov 2007.
[8] I.F.Akyyilidiz , w.Y Lee , K.R.chowdhary “ CRAHNs: cognitive Radio ad Hoc Networks" . Journal of Ad Hoc Networks, p 810-836, Science Direct, 2009.

[9] Ayman A. El-Saleh, Mahamod Ismail, Mohd. A. M. Ali, and Ahmed N. H. Alnuaimy "Capacity Optimization for Local and Cooperative Spectrum Sensing in Cognitive Radio Networks" World Academy of Science, Engineering and Technology 502009.

[10] Power Control in Cognitive Radio Systems Based on Spectrum Sensing Side Information Karama Hamdi, Wei Zhang, and Khaled Ben Letaief, Fellow, IEEE.

[11] A.A.El-Saleh, M.Ismail, M.A.M. Ali, A.N.H. Alnuaimy. "Capacity Optimization for Local and Cooperative Spectrum Sensing in Cognitive Radio Networks". Intern. Journal of Electronics, Circuits and Systems, Vol.3, 2009. 